\title{
Differentiation of basal cell carcinoma and healthy skin using multispectral modulation autofluorescence imaging: A pilot study
}

\author{
Nikita V. Chernomyrdin ${ }^{1,2 *}$, Anastasiya D. Lesnichaya ${ }^{2}$, Egor V. Yakovlev², Konstantin G. Kudrin ${ }^{3}$, \\ Olga P. Cherkasova ${ }^{4}$, Elena N. Rimskaya ${ }^{2,3}$, Vladimir N. Kurlov ${ }^{5}$, Valeriy E. Karasik2, \\ Igor V. Reshetov ${ }^{3}$, Valery V. Tuchin ${ }^{6,7,8}$, and Kirill I. Zaytsev ${ }^{1,2 \S}$ \\ ${ }^{1}$ Prokhorov General Physics Institute of the Russian Academy of Sciences, Moscow 119991, Russia \\ ${ }^{2}$ Bauman Moscow State Technical University, Moscow 105005, Russia \\ ${ }^{3}$ Sechenov University, Moscow 119991, Russia \\ ${ }^{4}$ Institute of Laser Physics of Siberian Branch of RAS, Novosibirsk 630090, Russia \\ ${ }^{5}$ Institute of Solid State Physics of RAS, Chernogolovka 142432, Moscow Obl., Russia \\ ${ }^{6}$ Saratov State University, Saratov 410012, Russia \\ 7 Tomsk State University, Tomsk 634050, Russia \\ ${ }^{8}$ Institute of Precision Mechanics and Control of the Russian Academy of Sciences, Saratov 410028, Russia \\ *e-mail: chernik-a@yandex.ru \\ § e-mail: kirzay@gmail.com
}

\begin{abstract}
An approach for differentiating basal cell carcinoma (BCC) and healthy skin by combining a multispectral modulation autofluorescence imaging with the linear discriminant analysis has been proposed. The experimental setup, which employs a 365-nm narrowband excitation, 4 replaceable bandpass filters and a digital camera, has been assembled and applied to study freshly excised samples of BCC. In the experimental setup, modulation of the UV-excitation and demodulation of the visible light images allow for both increasing a signal-to-noise ratio and suppressing a nonfluorescence background in the autofluorescence images of tissues. The observed results demonstrate an ability for distinguishing both ordinary and keratinized BCC from healthy skin justifying the perspectives of the multispectral modulation autofluorescence imaging use for non-invasive and intraoperative diagnosis of BCC and other low-pigmented malignancies of the skin. (C) 2019 Journal of Biomedical Photonics \& Engineering.
\end{abstract}

Keywords: multispectral fluorescence imaging; autofluorescence phenomenon; medical diagnosis; basal cell carcinoma; linear discriminant analysis.

Paper \#3309 received 17 Dec 2018; accepted for publication 20 Mar 2019; published online 27 Mar 2019. doi: 10.18287/JBPE19.05.010302.

\section{Introduction}

In the past decades, a rapid development of fluorescent spectroscopy, imaging and microscopy has been observed [1-3]. The list of fluorescence sensing techniques includes steady-state and time-resolved fluorescence spectroscopy [4], multi-spectral and hyperspectral imaging [5-10], two-photon fluorescence correlation spectroscopy and imaging [11-14], scattered-light fluorescence microscopy [15-18], etc.

Fluorescence spectra of biological tissues are formed by individual spectra of fluorophores [19]. By studying the shape of the fluorescence spectra or processing the data of multi-spectral fluorescence imaging, one could analyze the biochemical content and metabolic state of tissues, differentiate biological samples, and investigate 
its physiological stress sensitivity [4, 20,21]. Fluorescence spectroscopy and imaging are widely applied in biomedical science and for medical diagnosis in clinics $[1,22,23]$, including non-invasive, minimally-invasive and intraoperative diagnosis of cancers. The fluorescence imaging has been recently applied to diagnose malignancies of the colon [24-26], the esophagus [27-29], the gastric [30], the breast [31], the ovarian [32-34] and the brain [35-37].

One of the most promising applications of fluorescence spectroscopy and imaging is non-invasive and least-invasive diagnosis of skin cancers based on excitation either endogenous or exogenous fluorophores $[1,4,38]$. The methods of exogenous fluorescence diagnosis [39] employ various fluorescent dyes, which are injected into the skin, accumulated in cancer cells, fluoresce under a proper illumination, and, in some cases, could provide the photodynamic effect during the subsequent therapy [40-46]. This technique yields high sensitivity; however, it is also characterized by numerous disadvantages significantly limiting its reliability: (i) the procedure of dye delivery is rather time-consuming; (ii) it requires invasive delivery protocols (injection) or minimally-invasive topical or per-oral administration, and its further elimination from patient body; (iii) exogenous fluorescent dyes cannot provide satisfactory differentiation between similar pathologies (i.e. the specificity of the method is not high [4]).

The autofluorescence (AF) spectroscopy and imaging relying on endogenous fluorophores represent non-invasive and fast approach for skin cancers diagnosis. However, due to high pigmentation of pigmentary skin nevi [47] and early malignant melanomas of the skin $[48,49]$, this method has a significant limitation because of low light penetration depth [4]. The non-melanoma cancers of the skin [50], such as basal cell carcinoma (BCC) and squamous cell carcinoma (SCC), are usually less pigmented and, as a consequence, appear to be promising goal for the AF diagnosis [4, 51-58]. Despite the significant progress in this field of fluorescence biomedical technologies, the development of novel techniques for skin diagnosis based on AF remains a challenging problem.

In this paper, we propose an approach for differentiating BCC and healthy skin using the multispectral modulation AF imaging combined with linear discriminant analysis (LDA). We assemble an experimental setup employing a 365-nm narrowband excitation of the tissue sample AF, 4 replaceable bandpass filters and a digital camera. It allows for both increasing a signal-to-noise ratio and suppressing a nonfluorescent background in the AF images of tissues using modulation of the UV-excitation and demodulation of the AF visible light images. We use the experimental setup to study in vitro samples of freshly excised BCC from 6 patients. The results of this pilot study demonstrate ability for distinguishing ordinary and keratinized BCC from healthy skin using the proposed imaging approach. We analyze the accuracy of the tissue differentiation using this multispectral modulation AF imaging. We qualitatively compare the proposed technique with the existing developments in the area of multispectral AF imaging of BCC, which allows us to highlight the potentials of its use for noninvasive and intraoperative diagnosis of BCC and other low-pigmented skin malignancies.

\section{Experimental setup}

To study the images of non-melanoma skin cancer AF, we assembled a setup for the modulated multi-spectral fluorescence imaging $[59,60]$. It includes excitation and detection channels (see Fig. 1).

In the excitation channel, a polychromatic light of a mercury lamp radiates the sample of interest after passing a 365-nm narrowband excitation filter, a rectangular beam homogenizer, and a $f_{\text {mod }}=5 \mathrm{~Hz}$ mechanical chopper. The homogenizer shapes the UVbeam to rectangular form leading to the spatiallyhomogeneous irradiance of the sample, which is placed behind a non-fluorescent reference window (see Fig. 1(c)). Despite the polychromatic mercury lump with a total power of $100 \mathrm{~W}$ is employed in our study, a monochromatic UV-irradiance of the sample is only about $10 \mathrm{~mW} / \mathrm{cm}^{2}$ fulfilling the guidelines limiting an exposure of the skin in vivo to the electromagnetic waves [61], as specifically, the permissible UVirradiance of the skin is of about $100 \mathrm{~mW} / \mathrm{cm}^{2}$ for a typical measurement time of $10 \mathrm{sec}$.

The UV-radiation being interacted with tissue via scattering and emittance of fluorescence [62] is comprised of both the sharp line of the residual UVexcitation light and the broadband fluorescence varying for different regions of the sample under study. In the detection channel, a set of the bandpass interference filters and a digital camera are used for detection of the quasi-monochromatic visible light images $I_{\lambda}(t, \mathbf{x})$ of the object fluorescence, where index $\lambda$ defines a certain bandpass filter, $\mathbf{x}$ is the radius vector at the image plane, and $t$ is the time. Spectral transparency curves $T(\lambda)$ of the bandpass filters with different central wavelengths $\lambda \simeq 400,450,500$, and $550 \mathrm{~nm}$, respectively, and similar full-width at half-maximum (FWHM) of $\Delta \lambda \simeq 40 \mathrm{~nm}$ for all filters are shown in Fig. 1(b). We select the described set of the bandpass filters from commercially available ones to both almost continuously cover the most intense part of healthy skin and BCC AF spectra [4] and divide AF spectra into four initially-orthogonal ranges - i.e. separate channels of the multispectral imaging. We use a digital camera with complementary metal-oxide-semiconductor (CMOS) sensor and the frame rate of $f_{\text {rep }}=25 \mathrm{~Hz}$. 

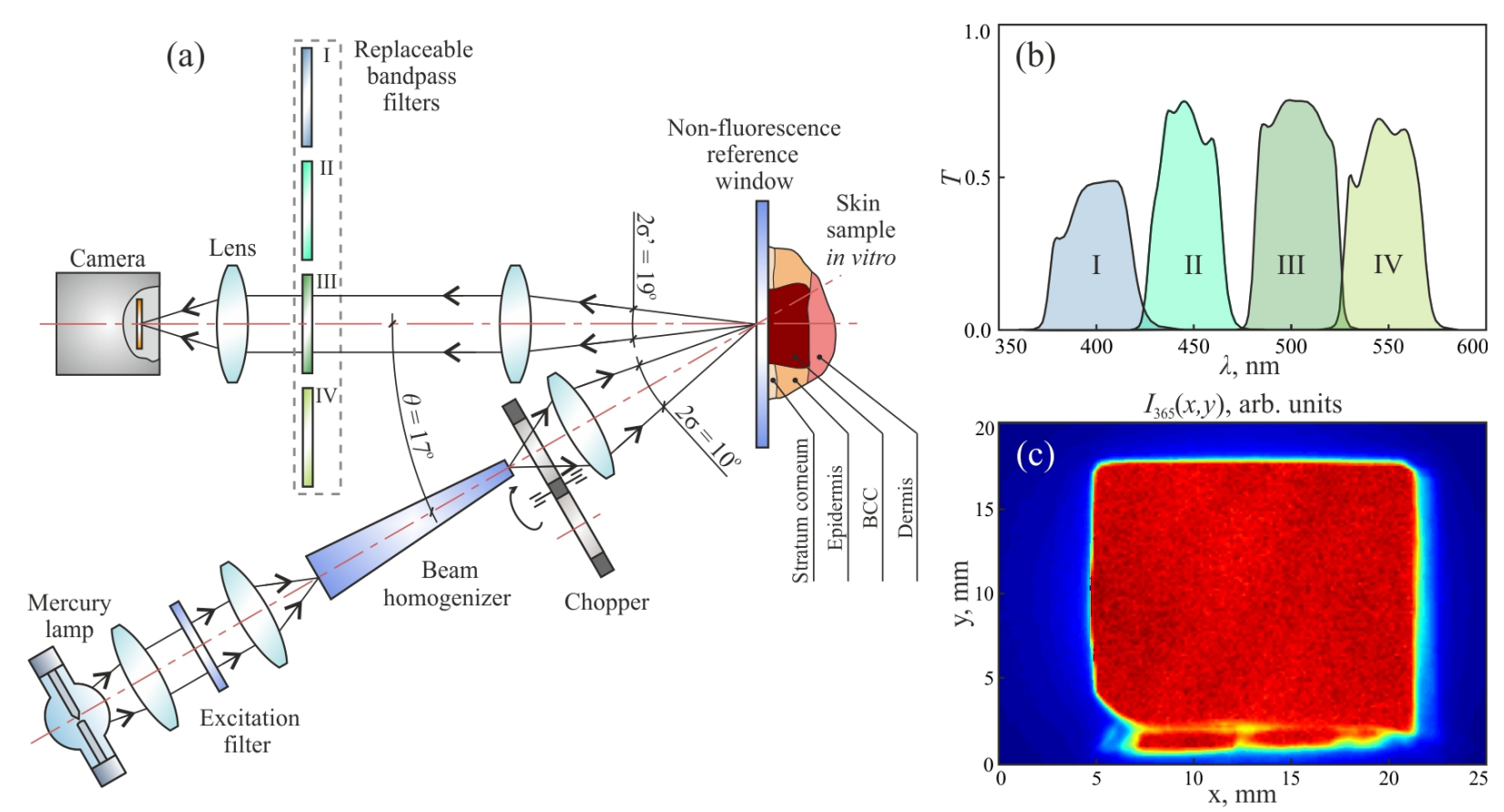

Fig. 1 An experimental setup for the multispectral modulated AF imaging: (a) a scheme of the experimental setup; (b) a spectral transmission of the optical bandpass filters in the detection channel; (c) a spatial distribution of the UVexcitation intensity at the object plane.

In the experimental setup (see Fig. 1), the average angle of the UV radiation incidence on the sample surface (i.e. the angle between the optical axis of the UV-excitation channel and the perpendicular to the sample surface) and the angle of the image detection (i.e. the angle between the optical axis of the imaging channel and the perpendicular to the sample surface) are $\theta \simeq 17^{\circ}$ and $\theta^{\prime} \simeq 0^{\circ}$, respectively. Numerical apertures of the UV-excitation and the fluorescence imaging system are $\mathrm{NA}=0.09\left(2 \sigma \simeq 10^{\circ}\right)$ and $\mathrm{NA}^{\prime}=0.16$ $\left(2 \sigma^{\prime} \simeq 19^{\circ}\right)$, respectively. Along with simplification of the experimental setup and minimization of its dimensions, the use of conventional low-aperture optics in excitation and detection channels allows for providing both a homogeneous irradiance of the sample surface and a spatial resolution of about $0.1 \mathrm{~mm}$ at the object plane for the entire field of view.

We perform fluorescence imaging in non-polarized light and assume isotropic character of the sample fluorescence indicatrix. This assumption is correct in case of the small apertures along with the close-tonormal angles of incidence and imaging [63]. At the same time, numerous researches, who are studying the fluorescence of turbid media (including, the fluorescence of tissues), have shown complex polarization-dependent and anisotropic character of this phenomenon [64-70]. By combining optimized geometry of fluorescence excitation and collection with modern fluorescence data processing techniques, one could mitigate an impact of the light scattering in a tissue, reconstruct its intrinsic fluorescence [71-75] and improve an efficiency of fluorescence collection [7678]. Furthermore, a polarization-dependent character and an anisotropy of tissue fluorescence can serve as a source of additional information for differentiating healthy and pathological tissues [79-81].

During the experimental study, the in vitro tissue sample is rigidly fixed behind the non-fluorescent reference window (see Fig. 1(a)). By rigidly fixing the sample surface towards the reference window, we eliminate the influence of various negative factors on the images: (i) an accuracy of the sample positioning; (ii) fluctuations of an incident angle of the UVradiation; and (iii) non-normal geometry of imaging (i.e. non-orthogonality of the imaging optical axis towards the sample surface). In our work, we consider lowpigmented malignancies of the skin - pigmentation of BCC is insignificant compared to highly-pigmented nevi and melanomas of the skin [4]. Therefore, we neglect the influence of a laterally-inhomogeneous tissue pigmentation on the data of the fluorescence imaging [82].

\section{Visible light image processing}

Using Fourier-domain analysis, we reconstruct the fluorescence images $f_{\lambda}(\mathrm{x})$ corresponding to various bandpass filters $\lambda[83]$ :

$$
I_{\lambda}(t, \mathbf{x}) \propto f_{\lambda}(\mathbf{x})\left(1+\sin \left(2 \pi f_{\bmod } t\right)\right)
$$

This technique employs modulation of the UVradiation intensity and demodulation of the image sequences in the visible range. It yields detecting a set of the sample AF images $f_{\lambda}(\mathbf{x})$ with both an increased signal-to-noise ratio and a reduced impact of the nonfluorescent background $[59,60]$. In order to provide accurate demodulation of the data, a frame rate of the 
imaging system $f_{\text {img }}$ should be much lower compared to the frame rate of the camera $f_{\text {rep }}$ and the modulation frequency $f_{\text {mod }}$. In the described experimental setup (see Fig. 1), the frame rate of the multispectral modulation $\mathrm{AF}$ imaging is about $f_{\mathrm{img}}=0.5 \mathrm{~Hz}$; it is primarily limited with the inertness of the CMOS sensor and could be further increased (even to real-time operation) by substituting the CMOS camera with the advanced one characterized with improved performance.

In order to study an ability for the differentiation of healthy and pathological tissues using the proposed multispectral AF imaging technique, we examined a set of freshly excised BCC samples provided by the Sechenov University (Moscow, Russia). The tissue samples came from 1 male and 5 female patients aged between 57 and 78 were investigated. The abnormal tissue specimens were investigated within 3 hours after the surgical removal. Classification of the normal skin and BCC tissues was done on the basis of initial clinical diagnosis and proved by a further histological examination of the biopsies. Table 1 summarizes the data on the BCC samples, including the final diagnosis, histological image, patient gender and age, side and size of excision.

Table 1 Data for in vitro BCC samples.

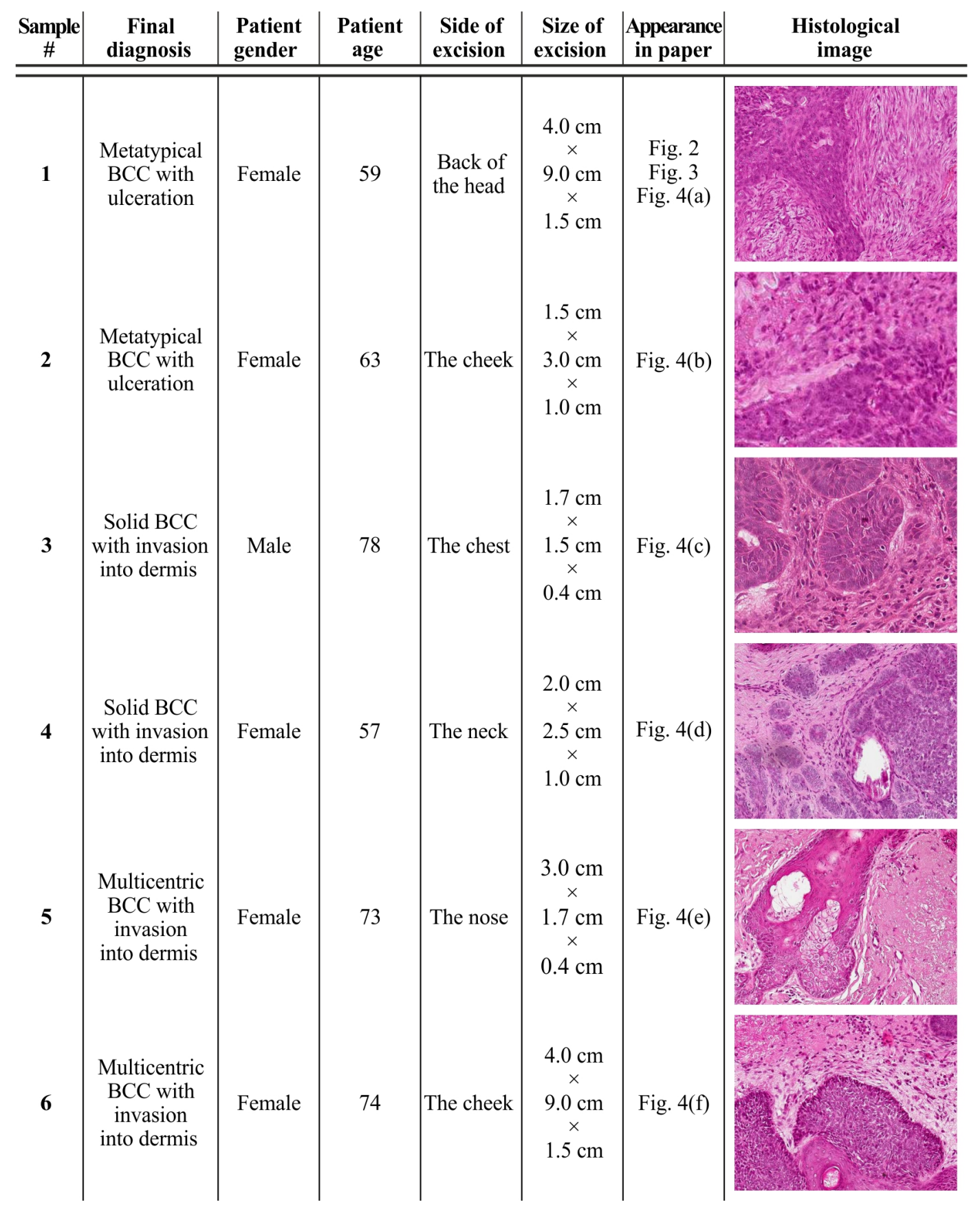


Figure 2 shows results of the multispectral modulation AF imaging of the $1^{\text {st }} \mathrm{BCC}$ sample from Table 1. Panel (a) shows digital image of the sample, where the borders mark the healthy skin (I), BCC (II), flake of keratinized BCC (III) and imaged area (IV). Panel (b) visualizes reverse contrast frames from AF image $I_{\lambda}(t, \mathbf{x})$ at $\lambda=500 \mathrm{~nm}$. Panel (c) shows a set of reverse contrast AF images $f_{\lambda}(\mathbf{x})$ of $\mathrm{BCC}$ extracted from the modulated AF sequences (see Eq. (1)). In complete accordance with the Refs. [53, 84], the experimental results show lower AF intensity for the ordinary BCC tissues. At the same time, the highest fluorescence intensity corresponds to the flake of keratinized BCC (region III in Fig. 2(a)).

The theoretical background for distinctive $\mathrm{AF}$ changes in cancer tissue was investigated earlier $[4,24,85,86]$. The altered metabolic state of cancer cells and resulting abnormal concentration of nicotinamide adenine dinucleotide (NADH) were suggested to be the origin of cancer AF changes $[86,87]$, which allows using the AF phenomenon for discriminating malignancies from normal tissues [8791]. The changes of the AF spectrum in malignant skin tissues (under the UV-excitation in the spectral range of 260 to $400 \mathrm{~nm}$ ) were reported to originate from the decreased collagen and elastin content in tissues, as well as from the decreased NADH levels [84, 92-98]. Furthermore, the differences in optical properties of healthy and pathological tissues (i.e. absorption and scattering of the UV-radiation and the fluorescence component) provide the contrast between the healthy skin and BCC in the AF spectra and images, which is originated owing to the listed features of malignant tissues.

A set of the four fluorescence images $f_{\lambda}(\mathbf{x})$ (see Fig. 2(c)) represents information about the sample AF intensity in different spectral channels. Such data could not be directly used for differentiation of tissues, for example, using simple intensity threshold, since AF intensity depends on a number of factors (the shape of the skin surface, its pigmentation and hydration, and others). For instance, according to the data of Fig. 2(c), the ordinary BCC tissue fluoresces less intensively comparing to the healthy skin; but, oppositely, the keratinized BCC fluoresces much intensively. Therefore, novel approach for tissue segmentation, which does not rely on simple AF intensity analysis should be introduced.

\section{LDA-based differentiation of tissues}

For differentiation of healthy and abnormal tissues, we perform statistical analysis of normalized fluorescence intensities in all of the four spectral channels using the LDA approach [93, 99] - i.e. a linear transformation technique, which yields reduction of the experimental data dimensionality and maximization of the separation between classes.
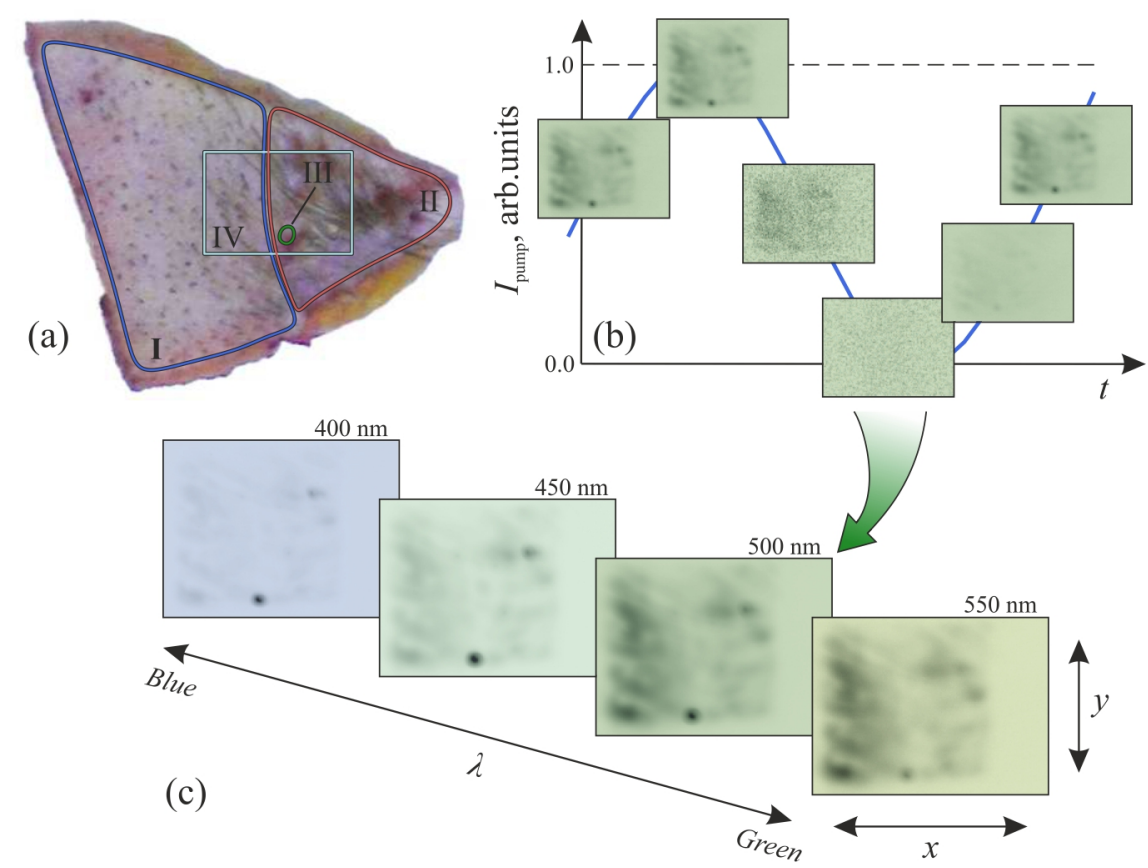

Fig. 2 A multispectral modulation AF imaging of BCC: (a) a clinical photo of the $1^{\text {st }}$ BCC sample (see Table 1), where characters I to IV define the healthy skin, BCC, flake of keratinized BCC and imaging areas, respectively; (b) reverse contrast frames of the multispectral AF image $I_{\lambda}(t, \mathbf{x})$ at $\lambda=500 \mathrm{~nm}$; (c) reverse contrast demodulated multispectral AF images $f_{\lambda}(\mathbf{x})$ at $\lambda=400,450,500,550 \mathrm{~nm}$. 
(a) Principal component space

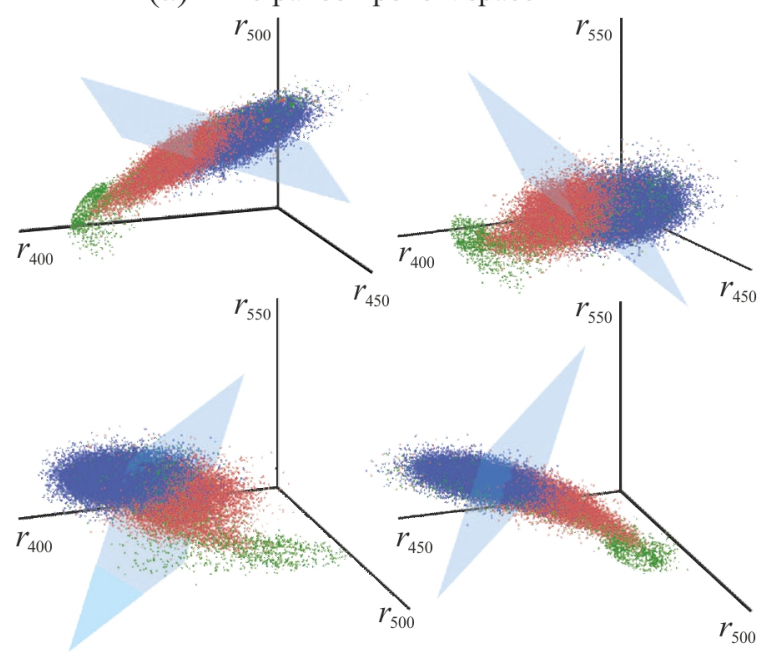

(b) Linear discriminant parameter

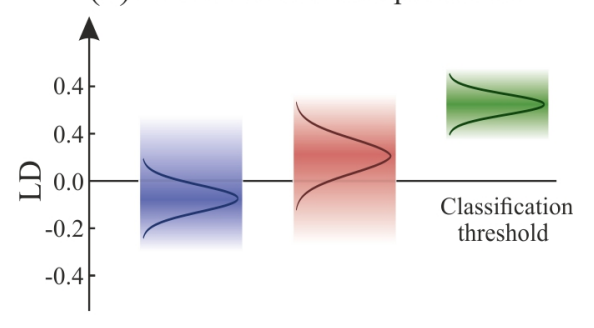

Healthy skin

BCC

Keratinized BCC

Fig. 3 A statistical analysis of the results of the multispectral modulation AF imaging: (a) a principal component space for the $1^{\text {st }}$ BCC sample (see Fig. 2 and Table 1); (b) LDA space for the $1^{\text {st }}$ BCC sample (see Fig. 2 and Table 1). Classes of the healthy skin, BCC and keratinized BCC are represented with blue, red and green areas, respectively. Light blue 4D plane in (a) and line in (b) show the threshold for discriminating healthy skin and BCC.

We introduce the principal components based on normalized intensities of the tissue AF. The intensities of each $\mathbf{x}_{i}$-pixel in the AF images $f_{\lambda}(\mathbf{x})$ define a radiusvector $\mathbf{r}_{i}$ in a four-dimensional (4D) principal component space

$$
\mathbf{r}_{i}=\left(\mathrm{r}_{400, i}, r_{450, i}, r_{500, i}, r_{550, i}\right)^{\mathrm{T}}
$$

where

$$
r_{\lambda, i}=\frac{f_{\lambda}\left(\mathbf{x}_{i}\right)}{\frac{1}{K} \sum_{n}^{K} f_{n}\left(\mathbf{x}_{i}\right)}
$$

is a normalized intensity corresponding to the $i^{\text {th }}$-pixel fluorescence in a certain spectral channel $n=400,450$, 500 or $550 \mathrm{~nm}$, and $K=4$ is a number of the spectral channels. These principal components are basically orthogonal due to initial orthogonality of the spectral transparency curves $T(\lambda)$ of the bandpass filters (see Fig. 1(b)). The full size of the AF image $f_{\lambda}$ is $M \times N$ points; thus, it allows for calculating $M \times N$ radius vectors in the principal component space.

Fig. 3(a) demonstrates an example of the principal component space, which is calculated based on the data of the $1^{\text {st }}$ BCC sample imaging (see Fig. 2 and Table 1). Namely, it shows three-dimensional (3D) projections of the four-dimensional principal component space, where various points are colored according to areas of the healthy skin (blue), BCC (red) and keratinized BCC (green). This data set represents a typical character of the principal component plot, which is observed for all BCC samples. The sets of points in the principal component space by BCC and healthy skin tissues form classes, which are principally distinguishable.

We have implemented the Fisher LDA approach [99] to highlight an ability for the differentiation of healthy skin and $\mathrm{BCC}$ based on the proposed $\mathrm{AF}$ imaging technique. In order to find the procedure of the data projection into the 1D Fisher LDA subspace and to estimate the threshold value, we perform the following data analysis.

- We perform multispectral modulation AF imaging of all six BCC samples (see Table 1).

- We manually choose a number of healthy skin and $\mathrm{BCC}$ points in the AF images $f_{\lambda}(\mathbf{x})$ in order to form healthy skin and BCC classes in the principal component space for further threshold selection. The amounts of healthy skin and BCC points, which are assigned a priori basing on the data of histological analysis and expert visual examination, varies from sample to sample. However, this amounts account at least 30 points for each tissue sample and each class.

- Using all the manually selected points, we form two groups of radius vectors $\mathbf{r}_{i, \text { skin }}, \mathbf{r}_{i, \mathrm{bcc}}$ representing healthy skin and BCC classes in the principal space. For these two classes, we calculate mean vectors $\langle\mathbf{r}\rangle_{\text {skin }},\langle\mathbf{r}\rangle_{\mathrm{bcc}}$ and scatterers $S_{\text {skin }}, S_{\mathrm{bcc}}$ :

$$
\begin{gathered}
\langle\mathbf{r}\rangle_{\mathrm{skin}}=\frac{1}{N_{\mathrm{skin}}} \sum_{i} \mathbf{r}_{i, \mathrm{skin}} ; \\
\langle\mathbf{r}\rangle_{\mathrm{bcc}}=\frac{1}{N_{\mathrm{bcc}}} \sum_{i} \mathbf{r}_{i, \mathrm{bcc}} ; \\
S_{\mathrm{skin}}=\frac{1}{N_{\mathrm{skin}}} \sum_{i}\left(\mathbf{r}_{i, \mathrm{skin}}-\langle\mathbf{r}\rangle_{\mathrm{skin}}\right)\left(\mathbf{r}_{i, \mathrm{skin}}-\langle\mathbf{r}\rangle_{\mathrm{skin}}\right)^{\mathrm{T}} ; \\
S_{\mathrm{bcc}}=\frac{1}{N_{\mathrm{bcc}}} \sum_{i}\left(\mathbf{r}_{i, \mathrm{bcc}}-\langle\mathbf{r}\rangle_{\mathrm{bcc}}\right)\left(\mathbf{r}_{i, \mathrm{bcc}}-\langle\mathbf{r}\rangle_{\mathrm{bcc}}\right)^{\mathrm{T}},
\end{gathered}
$$


where $N_{\text {skin }}$ and $N_{\text {bcc }}$ are numbers of priory selected points of healthy and abnormal tissues, respectively. Since LDA employs linear data projection into 1D subspace $\mu_{i}=\mathbf{w}^{\mathrm{T}} \mathbf{r}_{i}$ (here, $\mathbf{w}^{\mathrm{T}}$ is a projection operator), in 1D space we define the points of healthy skin and BCC classes $\mu_{i, \text { skin }}, \mu_{i \text {,bcc }}$, the related mean values $\langle\mu\rangle_{\text {skin }},\langle\mu\rangle_{\mathrm{bcc}}$ and the scatterers $s_{\text {skin }}, s_{\text {bcc }}$ :

$$
\begin{gathered}
\langle\mu\rangle_{\text {skin }}=\frac{1}{N_{\text {skin }}} \sum_{i} \mu_{i, \text { skin }} ; \\
\langle\mu\rangle_{\mathrm{bcc}}=\frac{1}{N_{\mathrm{bcc}}} \sum_{i} \mu_{i, \mathrm{bcc}} ; \\
s_{\text {skin }}^{2}=\frac{1}{N_{\mathrm{skin}}} \sum_{i}\left(\mu_{i, \mathrm{skin}}-\langle\mu\rangle_{\mathrm{skin}}\right)^{2} ; \\
s_{\mathrm{bcc}}^{2}=\frac{1}{N_{\mathrm{bcc}}} \sum_{i}\left(\mu_{i, \mathrm{bcc}}-\langle\mu\rangle_{\mathrm{bcc}}\right)^{2} .
\end{gathered}
$$

- The LDA should maximize separation of healthy skin and BCC classes in 1D subspace, and this is equal to maximizing the following criterion

$$
\begin{aligned}
F(\mathbf{w}) & =\frac{\left|\langle\mu\rangle_{\text {skin }}-\langle\mu\rangle_{\mathrm{bcc}}\right|^{2}}{s_{\text {skin }}^{2}+s_{\mathrm{bcc}}^{2}}= \\
& =\frac{\mathbf{w}^{\mathrm{T}} S_{\text {between }} \mathbf{w}}{\mathbf{w}^{\mathrm{T}} S_{\text {within }} \mathbf{w}}
\end{aligned}
$$

where

$$
S_{\text {between }}=\left(\langle\mathbf{r}\rangle_{\text {skin }}-\langle\mathbf{r}\rangle_{\text {bcc }}\right)\left(\langle\mathbf{r}\rangle_{\text {skin }}-\langle\mathbf{r}\rangle_{\mathrm{bcc}}\right)^{\mathrm{T}}
$$

and

$$
S_{\text {within }}=S_{\text {skin }}+S_{\text {bcc }}
$$

are between-class and within-class scatterers, respectively. By maximizing the criterion (4), we obtain the following solution for the projection operator

$$
\begin{aligned}
& \mathbf{w}^{\mathrm{T}}=\operatorname{argmax}[F(\mathbf{w})]= \\
& =S_{\text {within }}^{-1}\left(\langle\mathbf{r}\rangle_{\text {skin }}-\langle\mathbf{r}\rangle_{\text {bcc }}\right) .
\end{aligned}
$$

Thereby, by solving Eq. (5) with the priorycharacterize healthy skin and BCC points, we find the projection of the data to 1D Fisher LDA subspace.

- Knowing the mean values and the scatterers for the healthy and abnormal tissues, we set a threshold for the discrimination of these two classes in the LDA space. This threshold divides the space between the mean values $\langle\mu\rangle_{\text {skin }},\langle\mu\rangle_{\mathrm{bcc}}$ into two parts, in direct ratio to the values of scatterers $S_{\text {skin }}, S_{\mathrm{bcc}}$.
Figure 3(b) shows an LDA representation of the $1^{\text {st }}$ BCC sample measurements (see Table 1). The observed distributions of the data sets in the LDA space could be well-described by normal (Gaussian) probability density functions. Light blue line in Fig. 3(b) illustrates the value of the LDA threshold, which was determined using the described procedure based on the measurements of all six samples. In Fig. 3(a), equal linear threshold is shown as a light blue 4D plane in the principal component space. This threshold allows for differentiating both ordinary and keratinized BCC from healthy tissues of the skin.

After determining the projection operator $\mathbf{w}^{\mathrm{T}}$ and the threshold value, we could apply the following procedure to discriminate healthy and BCC tissue.

- Detection of the multispectral modulation AF images of the sample $I_{\lambda}(t, \mathbf{x})$. Demodulation of the AF images (see Eq. (1)) for obtaining the multispectral AF images $f_{\lambda}(\mathbf{x})$.

- Calculation of the experimental data representation in the principal component space (see Eqs. (2)-(3)) for the entire number of the image pixels.

- Projection of the data from the principal component space to the LDA space using the operator $\mathbf{w}^{\mathrm{T}}$ (see Eq. (5)).

- Differentiation of healthy skin and BCC tissues in the LDA space using the prior defined threshold value.

- $\quad$ Back-projection of the discriminated LDA data onto the initial visual image (clinical photo) of the sample.

In the LDA space (see Fig. 3(b)), both ordinary and keratinized BCC data appear to be shifted in one direction towards healthy skin data. The separation of tissues becomes possible because the LDA of the multispectral AF images allows studying spectrallydependent data on the tissue AF instead of analyzing only the AF intensity.

\section{LDA-Based Mapping of the Skin}

In order to highlight an ability for discrimination of BCC from healthy skin tissue using the proposed AF imaging approach, we implement equal projection operator $\mathbf{w}^{\mathrm{T}}$ and LDA threshold value for differentiating healthy and cancer tissues of all six BCC samples from Table 1. Figure 4 shows results of the tissue mapping: semi-transparent green masks represent the BCC area, which is estimated via the proposed multispectral AF imaging; red lines show the actual area of lesions. Since all of the imaged BCC samples correspond to the clinically identified type of BCC lesions (i.e. these samples do not contain any subsurface or clinically undetected BCC fragments), in our research, we estimate the actual area of the lesions by combining the visual examination of tissues by medical expert with the further histological study of tissue fragments.

Considering the results of the $1^{\text {st }}$ BCC sample mapping (see Fig. 4(a) and Table 1), we could observe 
high efficiency of the proposed technique. For this sample, we correctly identified $96.8 \%$ of BCC area and $85.7 \%$ of healthy skin area, while the mistakes of BCC and healthy skin identification were $3.2 \%$ and $14.3 \%$, respectively. For other BCC samples (panels from (b) to (f) in Fig. 4 corresponding to the BCC samples 2 to 6 in Table 1), we observed lower accuracy of tissue discrimination; Table 2 summarizes an analysis of the proposed technique accuracy, as well as reports averaged values $-76.4 \%$ and $82.5 \%$ of $\mathrm{BCC}$ and healthy skin tissues were accurately characterized. The minimal amount of imaged BCC samples is enough to highlight the ability for tissue differentiation using the proposed multispectral modulation AF imaging technique. However, this amount is not enough to perform in-depth statistical analysis of experimental data - i.e. to analyze sensitivity and specificity, type I and type II errors of tissue differentiation.

The accuracy of the tissue mapping could suffer from a number of factors, including laterallyinhomogeneous absorption [4, 82] and anisotropic polarization-dependent scattering $[64,73]$ of both the UV-radiation and the AF component in tissues. For further increase of imaging efficiency and tissue discrimination accuracy, the listed features of tissue should be taken into account during the analysis of the multispectral AF imaging data $[74,75,100]$.
Furthermore, by increasing the number of tissue samples at the stage of algorithm calibration, we could also significantly improve the proposed technique performance.

\section{Discussions}

The results of this pilot study involving in vitro tissue examination allow us to perform preliminary analysis, i.e. to perform feasibility test, which aims to objectively uncover strengths and weaknesses of the proposed AF imaging approach before committing to a full-blown study. Since physical properties of in vitro and in vivo tissues, including their AF spectra, could differ significantly depending on the time passed between surgery excision and experimental study, obviously, our future work should be dedicated to extensive clinical research focused on in vivo measurements. Nothing but the clinical research of in vivo samples, which involves the stage of method calibration based on large amount of samples and the stage of extensive statistical analysis of method performance, could form a basis for development of novel techniques for skin cancer diagnosis based on the multispectral modulation AF imaging.

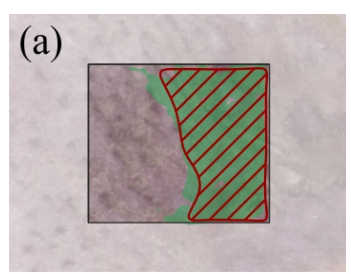

(d)

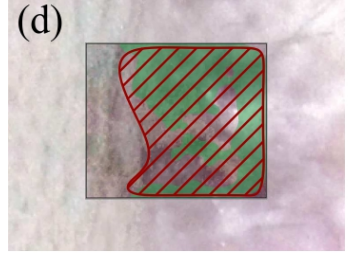

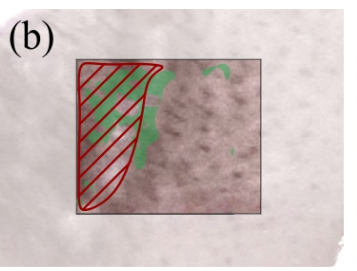

(e)

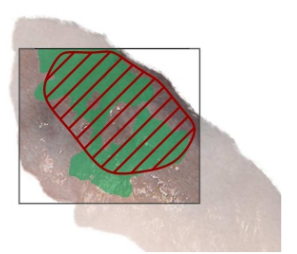

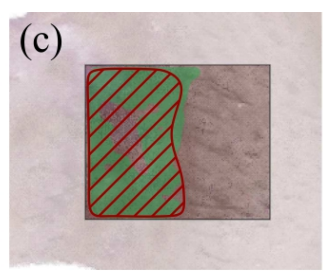

(f)

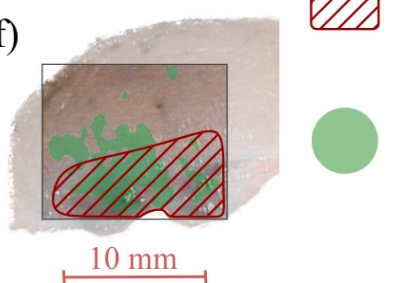

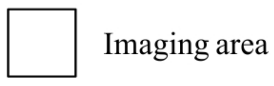

$\mathrm{BCC}$ area

(prior expert

examination

data)

$\mathrm{BCC}$ area

(fluorescence

imaging data)

Fig. 4 Mapping healthy and BCC tissue using the multi-spectral AF imaging: semi-transparent green masks represent the BCC area estimated via the proposed approach; red lines show the actual BCC area estimated via the visual examination of tissues by medical expert combined with the further histological study of tissues.

Table 2 Accuracy of healthy and abnormal tissue mapping.

\begin{tabular}{ccccccccc}
\hline Sample \# & 1 & 2 & 3 & 4 & 5 & 6 & Average & SD \\
\hline $\begin{array}{c}\text { Correctly identified } \\
\text { BCC tissue, } \%\end{array}$ & 96.8 & 50.7 & 81.3 & 71.6 & 88.2 & 70.1 & 76.4 & \pm 14.7 \\
\hline $\begin{array}{c}\text { Correctly identified } \\
\text { healthy skin, \% }\end{array}$ & 85.7 & 79.3 & 82.7 & 86.5 & 84.7 & 76.1 & 82.5 & \pm 3.7 \\
\hline
\end{tabular}


In our future research, we would also focus on the development of a portable experimental setup with improved operation rate, up to real-time imaging. For this purpose, we would: (i) increase the sensitivity of digital camera; (ii) optimize the UV-radiation parameters - i.e. select the optimal wavelength and increase the excitation power to the maximal permissible value; (iii) optimize a set of the bandpass optical filters for accommodating the spectrum of tissue $\mathrm{AF}$ and improving separability of classes of healthy and pathological tissues in the LDA-space; (iv) manage the LDA threshold value to maximize the tissue discrimination accuracy, and (v) use the advance methods of image processing to eliminate an impact of background tissue properties on differentiation accuracy. Other technical improvements, such as (i) substitution of the mercury lamp with UV-diode, (ii) increase of the number of UV-excitation channels to combine the AF imaging with the concept of fluorescent matrix [4], and (iii) improvement of the performance of optical systems in both excitation and imaging branches, can bring the system operation to a much higher level.

From a viewpoint of the spatial resolution and the examined object dimensions, all the existing fluorescence imaging methods can be divided into two classes - the methods of microscopic and macroscopic fluorescence imaging [3]. The microscopic techniques of the fluorescence imaging [84, 101-103] are characterized with very high lateral and depth spatial resolution, as well as advanced sensitivity, which yields visualizing the internal structure of the skin. These methods allow for studying separate cells and analyzing intravascular and interstitial cell processes. An AF microscopy was combined with Raman microspectroscopy for diagnosis of tissues and, in particular, for detection of BCC in tissue specimens excised during Mohs micrographic surgery $[104,105]$. Despite the tremendous resolution and sensitivity, microscopic fluorescence imaging techniques are characterized with limited field of view and excessive laboriousness, which makes them unreliable for use in a clinical practice.

The macroscopic fluorescence imaging techniques aim distinguishing between different types of tissues and finding their margins in a large fields of view and with a smaller (compared to microscopy) spatial resolution $[58,103,106]$. A modern trend in development of novel instruments for fluorescence diagnosis of the skin is associated with the use of multimodal imaging technologies - i.e combining endogenous and exogenous fluorescence phenomena [107] or combining fluorescence imaging with Raman micro-spectroscopy [108]. Furthermore, approaches of multispectral fluorescence imaging, which combine either several excitation and/or several detection channels with advanced methods of signal processing, are extensively developed during the past decades based on exogenous and endogenous fluorescence phenomena [106, 109-111].

The multispectral AF imaging approach proposed in this paper belongs to the group of the macroscopic fluorescence imaging techniques. Despite its technical simplicity, the proposed method provides relatively high performance, sensitivity and specificity of tissue differentiation compared to the existing methods of macroscopic AF diagnosis of low-pigmented skin malignancies [106, 109, 111]. The developed method allows for distinguishing both ordinary and keratinized $\mathrm{BCC}$ lesions from healthy skin thanks to the favorable combination of the narrow-line-width UV-excitation, the four orthogonal channels of image detection and the LDA-based approach for tissue discrimination, which relies on the use of normalized spectral AF intensities. The latter problem cannot be solved by simple analysis of the AF intensity since the AF intensity of ordinary $\mathrm{BCC}$ is lower, and the AF intensity of keratinized BCC is higher, compared to healthy skin [58]. The developed method provides discrimination of tissues even in case of simple low-aperture optical elements and standard uncooled CMOS-cameras are employed. This makes the proposed technique rather cheap; thus, more attractive and reliable for practical use in a clinic, when compared to the complex and expensive methods of the AF microscopy [84, 101-103] and the techniques, which combine fluorescence and Raman scattering phenomena [104, 105, 108].

In our opinion, the most promising application of the proposed multispectral AF imaging technique is associated with accomplishing the complete removal of the tumor and maximal preservation of the normal skin [112] by complementing such well-known techniques as Mohs micrographic surgery [113] and micrographic Doppler velocimetry [114], which are widely applied nowadays for removing BCC and SCC of the skin. In Mohs micrographic surgery, microscopic examination of all excised tissues occurs during the surgery, thereby providing tremendous accuracy and eliminating the need to estimate how far laterally or deep the cancer spreads [115]. In case of further increase of the described technique sensitivity, it could be applied in order to limit the amount of the tissue to be examined via microscopy during the cancer removal, and, as a consequence, to reduce the laboriousness and duration of the Mohs surgical procedure. Furthermore, this technique could be generalized for studying other types of biological objects, such as bacteria, or even for applications in pharmaceutical and material sciences, in chemical industry and food inspection.

\section{Conclusions}

In conclusion, in this paper, we proposed an approach for differentiating BCC and healthy skin, which relies on the use of the multispectral modulation $\mathrm{AF}$ imaging combined with the LDA-based processing of the normalized tissue AF intensities in four spectrallyorthogonal imaging channels. We enhanced the signalto-noise ratio and suppress the non-fluorescent background in the AF images by employing the modulation of the UV-radiation and the Fourier-domain demodulation of the AF visible images. We assembled the experimental setup and applied it for studying the 
samples of freshly excised BCC in vitro. The observed results demonstrate an ability for distinguishing both ordinary and keratinized BCC from healthy skin justifying the perspectives of the proposed technique use for diagnosis of $\mathrm{BCC}$ and other low-pigmented malignancies of the skin.

\section{Disclosures}

All authors declare that there is no conflict of interests in this paper.

\section{Acknowledgements}

Development of the approach for statistical analysis of the multispectral images, which yields differentiating healthy and pathological tissues, was supported by the grant of the Russian Foundation for Basic Research, Project \#18-29-02060. The authors are thankful to Prof. Larisa P. Safonova for fruitful discussions.

\section{References}

1. G. A. Wagnieres, W. M. Star, and B. C. Wilson, "In vivo fluorescence spectroscopy and imaging for oncological applications," Photochemistry and Photobiology 68(5), 603-632 (1998).

2. S. Andersson-Engels, C. af Klinteberg, K. Svanberg, and S. Svanberg, "In vivo fluorescence imaging for tissue diagnostics," Physics in Medicine \& Biology 42(5), 815-824 (1997).

3. R. Weissleder, M. J. Pittet, "Imaging in the era of molecular oncology," Nature 452(7187), 580-589 (2008).

4. E. G. Borisova, L. P. Angelova, and E. P. Pavlova, "Endogenous and exogenous fluorescence skin cancer diagnostics for clinical applications," IEEE Journal of Selected Topics in Quantum Electronics 20(2), 211-222 (2014).

5. I. Munro, J. McGinty, N. Galletly, J. Requejo-Isidro, P. M. P. Lanigan, D. S. Elson, C. Dunsby, M. A. A. Neil, M. J. Lever, G. W. H. Stamp, and P. M. W. French, "Toward the clinical application of time-domain fluorescence lifetime imaging," Journal of Biomedical Optics 10(5), 51403 (2005).

6. S. Shrestha, B. E. Applegate, J. Park, X. Xiao, P. Pande, and J. A. Jo, "High-speed multispectral fluorescence lifetime imaging implementation for in vivo applications," Optics Letters 35(15), 2558-2560 (2010).

7. F. Fereidouni, K. Reitsma, and H. C. Gerritsen, "High speed multispectral fluorescence lifetime imaging," Optics Express 21(10), 11769-11782 (2013).

8. O. Gutierrez-Navarro, D. U. Campos-Delgado, E. Arce-Santana, M. O. Mendez, and J. A. Jo, “A fully constrained optimization method for time-resolved multispectral fluorescence lifetime imaging microscopy data unmixing," IEEE Transactions on Biomedical Engineering 60(6), 1711-1720 (2013).

9. J. Bec, D. M. Ma, D. R. Yankelevich, J. Liu, W. T. Ferrier, J. Southard, and L. Marcu, "Multispectral fluorescence lifetime imaging system for intravascular diagnostics with ultrasound guidance: In vivo validation in swine arteries," Journal of Biophotonics 7(5), 281-285 (2014).

10. S. Cheng, R. M. Cuenca, B. Liu, B. H. Malik, J. M. Jabbour, K. C. Maitland, J. Wright, Y.-S. L. Cheng, and J. A. Jo, "Handheld multispectral fluorescence lifetime imaging system for in vivo applications," Biomedical Optics Express 5(3), 921-931 (2014).

11. E. L. Elson, D. Magde, "Fluorescence correlation spectroscopy. I. Conceptual basis and theory," Biopolymers 13(1), 1-27 (1974).

12. D. Magde, E. L. Elson, and W. W. Webb, "Fluorescence correlation spectroscopy. II. An experimental realization," Biopolymers 13(1), 29-61 (1974).

13. W. Denk, J. H. Strickler, and W. W. Webb, “Two-photon laser scanning fluorescence microscopy," Science 248(4951), 73-76 (1990).

14. K. M. Berland, P. T. C. So, and E. Gratton, "Two-photon fluorescence correlation spectroscopy: Method and application to the intracellular environment," Biophysical Journal 68(2), 694-701 (1995).

15. G. Tarrach, M. A. Bopp, D. Zeisel, and A. J. Meixner, "Design and construction of a versatile scanning near-field optical microscope for fluorescence imaging of single molecules," Review of Scientific Instruments 66(6), 35693575 (1995).

16. L. M. Miller, P. Dumas, N. Jamin, J.-L. Teillaud, J. Miklossy, and L. Forro, "Combining IR spectroscopy with fluorescence imaging in a single microscope: Biomedical applications using a synchrotron infrared source (invited)," Review of Scientific Instruments 73(3), 1357-1360 (2002).

17. H. Muramatsu, J. M. Kim, S. Sugiyama, and T. Ohtani, "Simultaneous multicolor fluorescence imaging by scanning near-field optical/atomic force microscopy," Review of Scientific Instruments 74(1), 100-103 (2003).

18. I. M. Vellekoop, C. M. Aegerter, "Scattered light fluorescence microscopy: Imaging through turbid layers," Optics Letters 35(8), 1245-1247 (2010).

19. J. W. Hastings, "Chemistries and colors of bioluminescent reactions: A review," Gene 173(1), 5-11 (1996). 
20. K. Nikolova, M. Zlatanov, T. Eftimov, D. Brabant, S. Yosifova, E. Halil, G. Antova, and M. Angelova, "Fluoresence spectra from vegetable oils using violet and blue LD/LED exitation and an optical fiber spectrometer," International Journal of Food Properties 17(6), 1211-1223 (2014).

21. E. V. Demidova, T. N. Goryachkovskaya, I. A. Mescheryakova, T. K. Malup, A. I. Semenov, N. A. Vinokurov, N. A. Kolchanov, V. M. Popik, and S. E. Peltek, "Impact of terahertz radiation on stress-sensitive genes of E.Coli cell," IEEE Transactions on Terahertz Science and Technology 6(3), 435-441 (2016).

22. H. J. C. M. Sterenborg, M. Motamedi, R. F. Wagner, M. Duvic, S. Thomsen, and S. L. Jacques, "In vivo fluorescence spectroscopy and imaging of human skin tumours," Lasers in Medical Science 9(3), 191-201 (1994).

23. A. N. Bashkatov, E. A. Genina, V. I. Kochubey, and V. V. Tuchin, "Optical properties of human skin, subcutaneous and mucous tissues in the wavelength range from 400 to $2000 \mathrm{~nm}$," Journal of Physics D: Applied Physics 38(15), 2543-2555 (2005).

24. K. T. Schomacker, J. K. Frisoli, C. C. Compton, T. J. Flotte, J. M. Richter, N. S. Nishioka, and T. F. Deutsch, "Ultraviolet laser-induced fluorescence of colonic tissue: Basic biology and diagnostic potential," Lasers in Medical Science 12(1), 63-78 (1992).

25. R. M. Cothren, M. V Sivak, J. Van Dam, R. E. Petras, M. Fitzmaurice, J. M. Crawford, J. Wu, J. F. Brennan, R. P. Rava, R. Manoharan, and M. S. Feld, "Detection of dysplasia at colonoscopy using laser-induced fluorescence: a blinded study," Gastrointestinal Endoscopy 44(2), 168-176 (2009).

26. T. E. Renkoski, B. Banerjee, L. R. Graves, N. S. Rial, S. A. H. Reid, V. L. Tsikitis, V. N. Nfonsam, P. Tiwari, H. Gavini, and U. Utzinger, "Ratio images and ultraviolet $\mathrm{C}$ excitation in autofluorescence imaging of neoplasms of the human colon," Journal of Biomedical Optics 18(1), 16005 (2013).

27. I. Georgakoudi, B. C. Jacobson, J. Van Dam, V. Backman, M. B. Wallace, M. G. Müller, Q. Zhang, K. Badizadegan, D. Sun, G. A. Thomas, L. T. Perelman, and M. S. Feld, "Fluorescence, reflectance, and lightscattering spectroscopy for evaluating dysplasia in patients with Barrett's esophagus," Gastroenterology 120(7), 1620-1629 (2001).

28. T. J. Pfefer, D. Y. Paithankar, J. M. Poneros, K. T. Schomacker, and N. S. Nishioka, "Temporally and spectrally resolved fluorescence spectroscopy for the detection of high grade dysplasia in Barrett's esophagus," Lasers in Surgery and Medicine 32(1), 10-16 (2003).

29. M. Kara, R. S. DaCosta, B. C. Wilson, N. E. Marcon, and J. Bergman, “Autofluorescence-based detection of early neoplasia in patients with Barrett's esophagus,” Digestive Diseases 22(2), 134-141 (2004).

30. M. Tatsuta, H. Iishi, M. Ichii, M. Baba, R. Yamamoto, S. Okuda, and K. Kikuchi, "Diagnosis of gastric cancers with fluorescein-labeled monoclonal antibodies to carcinoembryonic antigen," Lasers in Surgery and Medicine 9(4), 422-426 (1989).

31. V. G. Peters, D. R. Wyman, M. S. Patterson, and G. L. Frank, “Optical properties of normal and diseased human breast tissues in the visible and near infrared," Physics in Medicine \& Biology 35(9), 1317-1334 (1990).

32. S. D. Kamath, R. A. Bhat, S. Ray, and K. K. Mahato, "Autofluorescence of normal, benign, and malignant ovarian tissues: A pilot study," Photomedicine and Laser Surgery 27(2), 325-335 (2009).

33. T. E. Renkoski, K. D. Hatch, and U. Utzinger, "Wide-field spectral imaging of human ovary autofluorescence and oncologic diagnosis via previously collected probe data," Journal of Biomedical Optics 17(3), 36003 (2012).

34. R. George, M. Michaelides, M. A. Brewer, and U. Utzinger, "Parallel factor analysis of ovarian autofluorescence as a cancer diagnostic," Lasers in Surgery and Medicine 44(4), 282-295 (2012).

35. A. A. Potapov, S. A. Goryaynov, V. A. Okhlopkov, L. V. Shishkina, V. B. Loschenov, T. A. Savelieva, D. A. Golbin, A. P. Chumakova, M. F. Goldberg, M. D. Varyukhina, and A. Spallone, "Laser biospectroscopy and 5ALA fluorescence navigation as a helpful tool in the meningioma resection," Neurosurgical Review 39(3), 437447 (2016).

36. I. A. Shikunova, D. O. Stryukov, S. N. Rossolenko, A. M. Kiselev, and V. N. Kurlov, "Neurosurgery contact handheld probe based on sapphire shaped crystal," Journal of Crystal Growth 457, 265-269 (2017).

37. I. A. Shikunova, G. M. Katyba, K. I. Zaytsev, I. N. Dolganova, I. A. Shikunova, N. V. Chernomyrdin, S. O. Yurchenko, G. A. Komandin, I. V. Reshetov, V. V. Nesvizhevsky, and V. N. Kurlov, "Sapphire shaped crystals for waveguiding, sensing, and exposure applications," Progress in Crystal Growth and Characterization of Materials (2018).

38. M. Monici, "Cell and tissue autofluorescence research and diagnostic applications," Biotechnology Annual Review $11,227-256$ (2005).

39. F. Fischer, E. F. Dickson, J. C. Kennedy, and R. H. Pottier, “An affordable, portable fluorescence imaging device for skin lesion detection using a dual wavelength approach for image contrast enhancement and aminolaevulinic acid-induced protoporphyrin IX. Part II. In vivo testing," Lasers in Medical Science 16(3), 207-212 (2001).

40. B. Zhao, Y.-Y. He, "Recent advances in the prevention and treatment of skin cancer using photodynamic therapy," Expert Review of Anticancer Therapy 10(11), 1797-1809 (2010).

41. G. Terentyuk, E. Panfilova, V. Khanadeev, D. Chumakov, E. Genina, A. Bashkatov, V. Tuchin, A. Bucharskaya, G. Maslyakova, N. Khlebtsov, and B. Khlebtsov, "Gold nanorods with a hematoporphyrin-loaded silica shell for dual-modality photodynamic and photothermal treatment of tumors in vivo," Nano Research 7(3), 325-337 (2014). 
42. B. Khlebtsov, E. Tuchina, V. Tuchin, and N. Khlebtsov, "Multifunctional Au nanoclusters for targeted bioimaging and enhanced photodynamic inactivation of Staphylococcus aureus," RSC Advances 5(76), 61639-61649 (2015).

43. V. P. Zharov, E. I. Galanzha, E. V. Shashkov, N. G. Khlebtsov, and V. V. Tuchin., "In vivo photoacoustic flow cytometry for monitoring of circulating single cancer cells and contrast agents," Optics Letters 31(24), 3623 (2006).

44. J.-W. Kim, E. I. Galanzha, E. V. Shashkov, H.-M. Moon, and V. P. Zharov, "Golden carbon nanotubes as multimodal photoacoustic and photothermal high-contrast molecular agents," Nature Nanotechnology 4(10), 688694 (2009).

45. J. T. Alander, I. Kaartinen, A. Laakso, T. Pätilä, T. Spillmann, V. V. Tuchin, M. Venermo, and P. Välisuo, “A Review of indocyanine green fluorescent imaging in surgery," International Journal of Biomedical Imaging 2012, 940585 (2012).

46. E. I. Galanzha, R. Weingold, D. A. Nedosekin, M. Sarimollaoglu, J. Nolan, W. Harrington, A. S. Kuchyanov, R. G. Parkhomenko, F. Watanabe, Z. Nima, A. S. Biris, A. I. Plekhanov, M. I. Stockman, and V. P. Zharov, "Spaser as a biological probe," Nature Communications 8, 15528 (2017).

47. K. I. Zaytsev, K. G. Kudrin, V. E. Karasik, I. V. Reshetov, and S. O. Yurchenko, "In vivo terahertz spectroscopy of pigmentary skin nevi: Pilot study of non-invasive early diagnosis of dysplasia," Applied Physics Letters 106(5), 053702 (2015).

48. S. Andersson-Engels, R. Berg, K. Svanberg, and S. Svanberg, "Multi-colour fluorescence imaging in connection with photodynamic therapy of delta-amino levulinic acid (ALA) sensitised skin malignancies," Bioimaging 3(3), 134-143 (1995).

49. G. E. Pierard, C. Pierard-Franchimont, L. Dewalque, C. Charlier, T. Hermanns-Le, S. Pierard, and P. Delvenne, "In vivo skin fluorescence imaging in young Caucasian adults with early malignant melanomas," Clinical, Cosmetic and Investigational Dermatology 7, 225-230 (2014).

50. C. S. Joseph, R. Patel, V. A. Neel, R. H. Giles, and A. N. Yaroslavsky, "Imaging of ex vivo nonmelanoma skin cancers in the optical and terahertz spectral regions optical and terahertz skin cancers imaging," Journal of Biophotonics 7(5), 295-303 (2014).

51. R. Cubeddu, A. Pifferi, P. Taroni, A. Torricelli, G. Valentini, and E. Sorbellini, "Fluorescence lifetime imaging: an application to the detection of skin tumors," IEEE Journal of Selected Topics in Quantum Electronics 5(4), 923929 (1999).

52. A. M. Wennberg, F. Gudmundson, B. Stenquist, A. Ternesten, L. Mölne, A. Rosén, and O. Larkö, "In vivo detection of basal cell carcinoma using imaging spectroscopy," Acta Dermato-Venereologica 79(1), 54-61 (1999).

53. R. Na, I. M. Stender, and H. C. Wulf, "Can autofluorescence demarcate basal cell carcinoma from normal skin? A comparison with protoporphyrin IX fluorescence," Acta Dermato-Venereologica 81(4), 246-249 (2001).

54. A. N. Yaroslavsky, V. Neel, and R. R. Anderson, "Fluorescence polarization imaging for delineating nonmelanoma skin cancers," Optics Letters 29(17), 2010-2012 (2004).

55. R. Cicchi, D. Massi, S. Sestini, P. Carli, V. De Giorgi, T. Lotti, and F. S. Pavone, "Multidimensional non-linear laser imaging of Basal Cell Carcinoma," Optics Express 15(16), 10135-10148 (2007).

56. I. Kopriva, A. Peršin, H. Zorc, A. Pašić, J. Lipozenčić, K. Kostović, and M. Lončarić, "Visualization of basal cell carcinoma by fluorescence diagnosis and independent component analysis," Photodiagnosis and Photodynamic Therapy 4(3), 190-196 (2007).

57. T. Gambichler, G. Moussa, and P. Altmeyer, "A pilot study of fluorescence diagnosis of basal cell carcinoma using a digital flash light-based imaging system," Photodermatology, Photoimmunology \& Photomedicine 24(2), 67-71 (2008).

58. N. P. Galletly, J. McGinty, C. Dunsby, F. Teixeira, J. Requejo-Isidro, I. Munro, D. S. Elson, M. A. A. Neil, A. C. Chu, P. M. W. French, and G. W. Stamp, "Fluorescence lifetime imaging distinguishes basal cell carcinoma from surrounding uninvolved skin," British Journal of Dermatology 159(1), 152-161 (2008).

59. K. I. Zaytsev, A. V. Perchik, N. V. Chernomyrdin, K. G. Kudrin, I. V. Reshetov, and S. O. Yurchenko, "Hyperspectral modulation fluorescent imaging using double acousto-optical tunable filter based on TeO2 - crystals," Journal of Physics: Conference Series 584(1), 012017 (2015).

60. N. V. Chernomyrdin, K. I. Zaytsev, A. D. Lesnichaya, K. G. Kudrin, O. P. Cherkasova, V. N. Kurlov, I. A. Shikunova, A. V. Perchik, S. O. Yurchenko, and I. V. Reshetov, "Principle component analysis and linear discriminant analysis of multi-spectral autofluorescence imaging data for differentiating basal cell carcinoma and healthy skin," Proceeding of SPIE 9976, 99760B (2016).

61. G. Ziegelberger, "ICNIRP guidelines on limits of exposure to laser radiation of wavelengths between $180 \mathrm{~nm}$ and 1,000 um," Health Physics 105(3), 271-295 (2013).

62. V. V. Tuchin, Tissue Optics: Light Scattering Methods and Instruments for Medical Diagnostics, $3^{\text {rd }}$ ed., SPIE Press, Bellingham, USA (2015).

63. S. Holopainen, F. Manoocheri, and E. Ikonen, "Non-Lambertian behaviour of fluorescence emission from solid amorphous material," Metrologia 46(4), 197 (2009). 
64. M. Keijzer, R. R. Richards-Kortum, S. L. Jacques, and M. S. Feld, "Fluorescence spectroscopy of turbid media: Autofluorescence of the human aorta," Applied Optics 28(20), 4286 (1989).

65. J. Wu, F. Partovi, M. S. Field, and R. P. Rava, "Diffuse reflectance from turbid media: an analytical model of photon migration," Applied Optics 32(7), 1115 (1993).

66. A. J. Durkin, S. Jaikumar, N. Ramanujam, and R. Richards-Kortum, "Relation between fluorescence spectra of dilute and turbid samples," Applied Optics 33(3), 414 (1994).

67. M. A. O’Leary, D. A. Boas, X. D. Li, B. Chance, and A. G. Yodh, "Fluorescence lifetime imaging in turbid media," Optics Letters 21(2), 158 (1996).

68. B. B. Das, F. Liu, and R. R. Alfano, "Time-resolved fluorescence and photon migration studies in biomedical and model random media," Reports on Progress in Physics 60(2), 227-292 (1997).

69. S. C. Gebhart, A. Mahadevan-Jansen, and W.-C. Lin, "Experimental and simulated angular profiles of fluorescence and diffuse reflectance emission from turbid media," Applied Optics 44(23), 4884 (2005).

70. L. G. Coppel, N. Johansson, and M. Neuman, "Angular dependence of fluorescence from turbid media," Optics Express 23(15), 19552 (2015).

71. J. Wu, M. S. Feld, and R. P. Rava, “Analytical model for extracting intrinsic fluorescence in turbid media,” Applied Optics 32(19), 3585 (1993).

72. C. M. Gardner, S. L. Jacques, and A. J. Welch, "Fluorescence spectroscopy of tissue: recovery of intrinsic fluorescence from measured fluorescence," Applied Optics 35(10), 1780 (1996).

73. J. Y. Qu, Z. Huang, and J. Hua, "Excitation-and-collection geometry insensitive fluorescence imaging of tissuesimulating turbid media," Applied Optics 39(19), 3344 (2000).

74. Q. Zhang, M. G. Müller, J. Wu, and M. S. Feld, "Turbidity-free fluorescence spectroscopy of biological tissue," Optics Letters 25(19), 1451 (2000).

75. M. G. Müller, I. Georgakoudi, Q. Zhang, J. Wu, and M. S. Feld, "Intrinsic fluorescence spectroscopy in turbid media: disentangling effects of scattering and absorption," Applied Optics 40(25), 4633 (2001).

76. K. Koyama, M. Yoshita, M. Baba, T. Suemoto, and H. Akiyama, "High collection efficiency in fluorescence microscopy with a solid immersion lens,” Applied Physics Letters 75(12), 1667-1669 (1999).

77. C. A. Combs, A. Smirnov, D. Chess, D. B. Mcgavern, J. L. Schroeder, J. Riley, S. S. Kang, M. Lugar-Hammer, A. Gandjbakhche, J. R. Knutson, and R. S. Balaban, "Optimizing multiphoton fluorescence microscopy light collection from living tissue by noncontact total emission detection (epiTED), "Journal of Microscopy 241(2), 153161 (2011).

78. J. P. Zinter, M. J. Levene, "Maximizing fluorescence collection efficiency in multiphoton microscopy," Optics Express 19(16), 15348 (2011).

79. T. Ruckstuhl, D. Verdes, "Supercritical angle fluorescence (SAF) microscopy," Optics Express 12(18), 4246 (2004).

80. J. J. Fisz, "Fluorescence polarization spectroscopy at combined high-aperture excitation and detection: Application to one-photon-excitation fluorescence microscopy," The Journal of Physical Chemistry A 111(35), 8606-8621 (2007).

81. J. J. Fisz, "Another treatment of fluorescence polarization microspectroscopy and imaging," The Journal of Physical Chemistry A 113(15), 3505-3516 (2009).

82. A. Curtis, K. Calabro, J.-R. Galarneau, I. J. Bigio, and T. Krucker, "Temporal Variations of Skin Pigmentation in C57Bl/6 Mice Affect Optical Bioluminescence Quantitation," Molecular Imaging and Biology 13(6), 1114-1123 (2011).

83. J. R. Lakowicz (ed), Principles of Fluorescence Spectroscopy, Springer, Boston, MA, USA (2006).

84. E. Drakaki, C. Dessinioti, A. J. Stratigos, C. Salavastru, and C. Antoniou, "Laser-induced fluorescence made simple: implications for the diagnosis and follow-up monitoring of basal cell carcinoma," Journal of Biomedical Optics 19(3), 30901 (2014).

85. E. Mitrani, R. Marks, "Procollagen localisation in normal, premalignant and malignant lesions of the epidermis," Archives of Dermatological Research 274(1), 21-28 (1982).

86. J. C. Zhang, H. E. Savage, P. G. Sacks, T. Delohery, R. R. Alfano, A. Katz, and S. P. Schantz, "Innate cellular fluorescence reflects alterations in cellular proliferation," Lasers in Surgery and Medicine 20(3), 319-331 (1997).

87. W. Lohmann, M. Nilles, and R. H. Bodeker, "In situ differentiation between nevi and malignant melanomas by fluorescence measurements," Naturwissenschaften 78(10), 456-457 (1991).

88. N. Ramanujam, M. F. Mitchell, A. Mahadevan, S. Warren, S. Thomsen, E. Silva, and R. Richards-Kortum, "In vivo diagnosis of cervical intraepithelial neoplasia using 337-nm-excited laser-induced fluorescence," Proceedings of the National Academy of Sciences 91(21), 10193-10197 (1994).

89. F. Koenig, F. J. McGovern, A. F. Althausen, T. F. Deutsch, and K. T. Schomacker, "Laser induced autofluorescence diagnosis of bladder cancer," Journal of Urology 156(5), 1597-1601 (1996).

90. M. Zellweger, D. Goujon, R. Conde, M. Forrer, H. van den Bergh, and G. Wagnières, “Absolute autofluorescence spectra of human healthy, metaplastic, and early cancerous bronchial tissue in vivo," Applied Optics 40(22), 37843791 (2001). 
91. E. Borisova, P. Troyanova, P. Pavlova, and L. Avramov, "Diagnostics of pigmented skin tumors based on laserinduced autofluorescence and diffuse reflectance spectroscopy," Quantum Electronics 38(6), 597 (2008).

92. Q. Liu, "Role of optical spectroscopy using endogenous contrasts in clinical cancer diagnosis," World Journal of Clinical Oncology 2(1), 50-63 (2011).

93. G. McLachlan, Discriminant Analysis and Statistical Pattern Recognition, Wiley, New York, USA (2004).

94. R. R. Anderson, J. A. Parrish, “The optics of human skin," Journal of Investigative Dermatology 77(1), 13-19 (1981).

95. Z. Volynskaya, A. S. Haka, K. L. Bechtel, M. Fitzmaurice, R. Shenk, N. Wang, J. Nazemi, R. R. Dasari, and M. S. Feld, "Diagnosing breast cancer using diffuse reflectance spectroscopy and intrinsic fluorescence spectroscopy," Journal of Biomedical Optics 13(2), 24012 (2008).

96. I. Bliznakova, E. Borisova, and L. Avramov, "Laser- and Light-Induced Autofluorescence Spectroscopy of Human Skin in Dependence on Excitation Wavelengths," Acta Physica Polonica A 112(5), 1131-1136 (2007).

97. E. Borisova, P. Pavlova, E. Pavlova, P. Troyanova, and L. Avramov, "Optical biopsy of human skin - A tool for cutaneous tumours' diagnosis," International Journal Bioautomation 16(1), 53-72 (2012).

98. R. Richards-Kortum, R. P. Rava, R. E. Petras, M. Fitzmaurice, M. Sivak, and M. S. Feld, "Spectroscopic diagnosis of colonic dysplasia," Photochemistry and Photobiology 53(6), 777-786 (1991).

99. R. A. Fischer, "The use of multiple measurements in taxonomic problems," Annals of Eugenics 7(2), 179-188 (1936).

100.R. Na, I.-M. Stender, M. Henriksen, and H. C. Wulf., "Autofluorescence of human skin is age-related after correction for skin pigmentation and redness," Journal of Investigative Dermatology 116(4), 536-540 (2001).

101.J. Paoli, M. Smedh, A.-M. Wennberg, and M. B. Ericson, "Multiphoton laser scanning microscopy on nonmelanoma skin cancer: morphologic features for future non-invasive diagnostics," Journal of Investigative Dermatology 128(5), 1248-1255 (2008).

102.R. Patalay, C. Talbot, Y. Alexandrov, I. Munro, M. A. A. Neil, K. König, P. M. W. French, A. Chu, G. W. Stamp, and C. Dunsby, "Quantification of cellular autofluorescence of human skin using multiphoton tomography and fluorescence lifetime imaging in two spectral detection channels," Biomedical Optics Express 2(12), 3295 (2011).

103.D. Göppner, N. Mechow, J. Liebscher, E. Thiel, G. Seewald, H. Gollnick, C. M. Philipp, and K.-H. Schönborn, "Wide-field, high-resolution two-photon tissue mapping of human skin ex vivo," Medical Laser Application 26(4), $158-165$ (2011).

104.K. Kong, C. J. Rowlands, S. Varma, W. Perkins, I. H. Leach, A. A. Koloydenko, H. C. Williams, and I. Notingher, "Diagnosis of tumors during tissue-conserving surgery with integrated autofluorescence and Raman scattering microscopy," Proceedings of the National Academy of Sciences 110(38), 15189-15194 (2013).

105.S. Takamori, K. Kong, S. Varma, I. Leach, H. C. Williams, and I. Notingher, "Optimization of multimodal spectral imaging for assessment of resection margins during Mohs micrographic surgery for basal cell carcinoma," Biomedical Optics Express 6(1), 98 (2015).

106.A. Lihachev, A. Derjabo, I. Ferulova, M. Lange, I. Lihacova, and J. Spigulis, "Autofluorescence imaging of basal cell carcinoma by smartphone RGB camera," Journal of Biomedical Optics 20(12), 120502 (2015).

107.M. B. Ericson, C. Berndtsson, B. Stenquist, A.-M. Wennberg, O. Larko, and A. Rosen, "Multispectral fluorescence imaging of basal cell carcinoma assisted by image warping," Proceeding of SPIE 5141, 114-121 (2003).

108.F. Sinjab, K. Kong, G. Gibson, S. Varma, H. Williams, M. Padgett, and I. Notingher, "Tissue diagnosis using power-sharing multifocal Raman micro-spectroscopy and auto-fluorescence imaging," Biomedical Optics Express 7(8), 2993 (2016).

109.B. Stenquist, M. B. Ericson, C. Strandeberg, L. Molne, A. Rosen, O. Larko, and A. M. Wennberg, "Bispectral fluorescence imaging of aggressive basal cell carcinoma combined with histopathological mapping: a preliminary study indicating a possible adjunct to Mohs micrographic surgery,” British Journal of Dermatology 154(2), 305309 (2006).

110.F. Fischer, E. F. Gudgin Dickson, and R. H. Pottier, "In vivo fluorescence imaging using two excitation and/or emission wavelengths for image contrast enhancement," Vibrational Spectroscopy 30(2), 131-137 (2002).

111.M. B. Ericson, J. Uhre, C. Strandeberg, B. Stenquist, O. Larkö, A.-M. Wennberg, and A. Rosén, "Bispectral fluorescence imaging combined with texture analysis and linear discrimination for correlation with histopathologic extent of basal cell carcinoma," Journal of Biomedical Optics 10(3), 034009 (2005).

112.N. Zhu, C.-Y. Huang, S. Mondal, S. Gao, C. Huang, V. Gruev, S. Achilefu, and R. Liang, "Compact wearable dualmode imaging system for real-time fluorescence image-guided surgery," Journal of Biomedical Optics 20(9), 96010 (2015).

113.R. Chuong, "Mohs surgery. Techniques, indications, applications, and the future," Journal of Oral and Maxillofacial Surgery 42(4), 274 (1984).

114.R. S. Kirsner, M. Haiken, and L. D. Garland, "Margin assessment of selected basal cell carcinomas utilizing laser doppler velocimetry," International Journal of Dermatology 32(4), 290-292 (1993).

115.G. R. Mikhail, Mohs Micrographic Surgery, W.B. Saunders, Philadelphia, PA, USA (1991). 